\title{
High continuous wave output power InGaAs/InGaAsP/InGaP diode lasers: Effect of substrate misorientation
}

\author{
L. J. Mawst,, a) A. Bhattacharya, M. Nesnidal, J. Lopez, and D. Botez \\ ECE Department, University of Wisconsin-Madison, Madison, Wisconsin 53706-1691
}

J. A. Morris

LDX Optronics, Orlando, Florida 32809

P. Zory

University of Florida, Gainesville, Florida 32611

$3 \mathrm{~W} \mathrm{cw}$ output power has been obtained from aluminum-free, strained-layer double-quantum well (DQW) InGaAs/InGaAsP/InGaP uncoated, $100-\mu \mathrm{m}$-wide stripe diode lasers $(\lambda=0.945 \mu \mathrm{m})$ grown by low-pressure MOCVD on exact (100) GaAs substrates. The combination of high-band-gap (1.62 $\mathrm{eV}$ ) InGaAsP confinement layers and the DQW structure provides relatively weak temperature dependence for both the threshold current $I_{\text {th }}$ as well as the external differential quantum efficiency $\eta_{d}$. Furthermore, the series electrical resistance for $100 \mu \mathrm{m} \times 600 \mu \mathrm{m}$ stripe-contact devices is as low as $0.12 \Omega$. As a result, the power conversion efficiency reaches a maximum of $40 \%$ at 8 $\times I_{\text {th }}$, and decreases to only $33 \%$ at the maximum power (i.e., $3 \mathrm{~W}$ ) at $28 \times I_{\text {th. }}$. Low-temperature (12 $\mathrm{K})$ photoluminescence measurements of InGaAs/InGaAsP quantum-well structures exhibit narrow linewidths $(<10 \mathrm{meV})$ for material grown on exact (100) GaAs substrates, while growths on misoriented substrates exhibit linewidth broadening, as a result of "step bunching." Laser structures grown on misoriented substrates exhibit increased temperature sensitivity of both $I_{\text {th }}$ and $\eta_{d}$, compared with structures grown on exact (100) substrates. () 1995 American Institute of Physics.

Strained-layer quantum-well InGaAs lasers $(980 \mathrm{~nm})$ are needed as high-output-power pump sources for Er-doped fiber and waveguide amplifiers as well as for fluoride-fiberbased frequency upconversion. The use of the aluminum-free InGaAs/InGaAsP/InGaP material system has several advantages over the InGaAs/GaAs/AlGaAs material system for the realization of reliable, high-power diode sources: (1) the low reactivity of InGaP to oxygen facilitates regrowth for the fabrication of single-mode index-guided structures, ${ }^{1,2}$ (2) higher electrical and thermal conductivity compared with $\mathrm{AlGaAs},{ }^{3}$ and (3) lasers with $\mathrm{AlGaAs}$ cladding layers and unpassivated facets show higher facet degradation than similar lasers with InGaP cladding layers, ${ }^{4}$ presumably due to the lower surface recombination velocity of $\mathrm{InGaP}^{5}$ compared with AlGaAs. Al-free lasers also exhibit an order of magnitude lower facet temperature rise compared with devices containing $\mathrm{AlGaAs}$ in both the confining and cladding layers. ${ }^{6}$ Although high performance has been demonstrated from Al-free laser structures, the characterization of the quantum-well growth for this material system and its influence on device performance have not been established.

Achieving high cw output power requires weak temperature dependence of both the threshold current, $I_{\text {th }}$, and differential quantum efficiency, $\eta_{d}$ which in turn are influenced by carrier leakage from the quantum well(s). Al-free lasers with GaAs or low-band-gap InGaAsP confinement layers exhibit strong carrier leakage from the InGaAs quantum well to the (optical) confinement layers, resulting in a strong temperature dependence of both $I_{\text {th }}$ and $\eta_{d}{ }^{7-9}$ To circumvent this problem, the use of higher band-gap InGaAsP confinement layers and multiple quantum wells have been used to

\footnotetext{
${ }^{a)}$ Electronic mail: mawst@engr.wisc.edu
}

reduce carrier leakage, and thereby reduce temperature sensitivity. ${ }^{8-11}$ Optimization of such laser structures requires an understanding of the nature of quantum-well growth for the InGaAs/InGaAsP/InGaP material system.

Recently, Hiramoto et al. ${ }^{12}$ have shown that the photoluminescence (PL) intensity and linewidth of $\mathrm{InGaAs} / \mathrm{GaAs}$ quantum-well (QW) structures grown by MOCVD is strongly dependent on substrate misorientation, due to stepbunching effects on vicinal substrates. However, no study has been undertaken on the influence of substrate misorientation on the optical properties of InGaAs/InGaAsP/InGaP QW structures grown by MOCVD on GaAs substrates. Here we report on the effects of substrate misorientation on the optical characteristics of strained-layer InGaAs QW structures and lasers with $\mathrm{InGaAsP}$ confinement layers lattice matched to GaAs substrates with nominally exact (100) $\pm 0.1^{\circ}$ orientation, $2^{\circ}, 6^{\circ}$, and $10^{\circ}$ misoriented towards [110], and $10^{\circ}$ misoriented towards [111]A. Low-temperature PL and diode-laser device performance indicate that optimal QW growth occurs for on-orientation (100) substrates. (These findings are in marked contrast to the optimal growth conditions of InGaP/AlGaInP quantum wells for visible lasers, which are typically grown on highly misoriented GaAs substrates. $\left.{ }^{13,14}\right)$ Broad-area, uncoated diode lasers (100 $\mu \mathrm{m}$ $\times 600 \mu \mathrm{m})$ fabricated from double-quantum-well (DQW) material grown on exact (100) GaAs substrates have achieved $3 \mathrm{~W}$ cw power, which represents the highest output power to date reported from $\mathrm{Al}$-free $\mathrm{InGaAs} / \mathrm{InGaAsP} / \mathrm{InGaP}$ $(\lambda=0.94-0.98 \mu \mathrm{m})$ diode lasers.

Single QW InGaAs structures with InGaAsP $(1.62 \mathrm{eV})$ barriers were used for low-temperature PL studies. The structures are grown by low pressure $(50 \mathrm{mbar}) \mathrm{MOCVD}$ in an Aixtron A-200 system at a temperature of $700{ }^{\circ} \mathrm{C}$. The V/III ratio for the growth of InGaP, InGaAsP, and InGaAs layers 


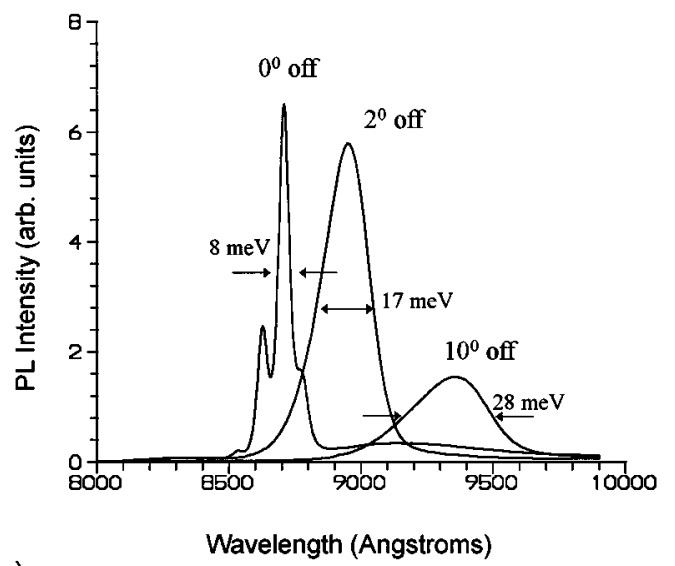

(a)

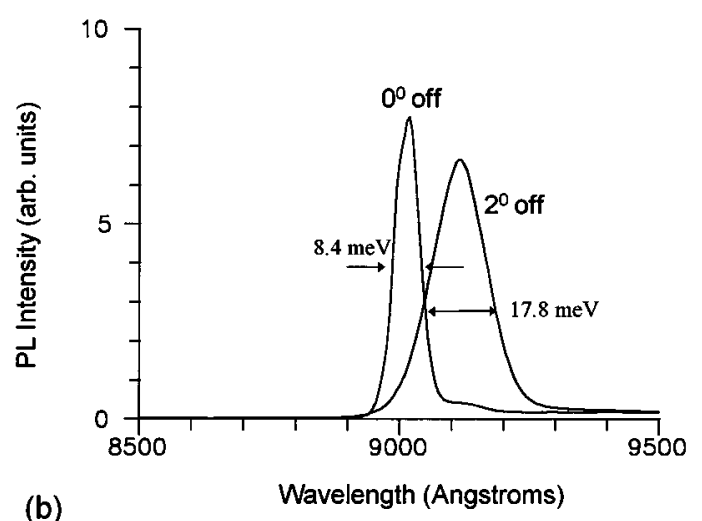

FIG. 1. Low-temperature $(12 \mathrm{~K})$ photoluminescence of (a) $3.5 \mathrm{~nm}$ wide $\operatorname{In}_{x} \mathrm{Ga}_{1-x}$ As $(x=0.18)$ with InGaAsP $\left(E_{g}=1.62 \mathrm{eV}\right)$ barriers grown on (100) GaAs substrates $0^{\circ}, 2^{\circ}$, and $10^{\circ}$ off towards [110], and (b) $7.0 \mathrm{~nm}$ wide $\operatorname{In}_{x} \mathrm{Ga}_{1-x}$ As $(x=0.18)$ with $\operatorname{InGaAsP}\left(E_{g}=1.62 \mathrm{eV}\right)$ barriers grown on (100) GaAs substrates $0^{\circ}$-, $2^{\circ}$-off towards [110]. Narrow linewidths are obtained only for growths on exact (100) substrates.

are 200,130 , and 100 , respectively. The interrupt times for switching between layers in the quantum wells were optimized to $2.5 \mathrm{~s}$ in order to obtain the highest PL intensity. Low-temperature (12 K) PL from QW structures with $3.5 \mathrm{~nm}$ wide $\operatorname{In}_{x} \mathrm{Ga}_{1-x}$ As $(x=0.18, \Delta a / a=0.013)$ layer are shown in Fig. 1(a), for growth on exact (100), $2^{\circ}$, and $10^{\circ}$ off towards [110] substrates, and in Fig. 1(b) for a $7.0 \mathrm{~nm}$ wide $\mathrm{In}_{x} \mathrm{Ga}_{1-x}$ As $(x=0.18)$ quantum well grown on exact (100) and $2^{\circ}$ towards [110]. Narrow linewidth $(<10 \mathrm{meV})$ multiplet-type luminescence are obtained only for growth on exact (100) GaAs substrates. The multiple emission peaks observed for on-orientation substrate growths are similar to features previously observed in the PL emission from InGaAs quantum wells on InP substrates, which has been attributed to lateral monolayer thickness variations in the quantum wells. ${ }^{15}$ By contrast, growths on misoriented substrates exhibit broadened luminescence, shifted towards longer wavelength. Similar broadening was also observed for growths occurring on (100) substrates orientated $10^{\circ}$ towards [111] A. The broadening of the PL linewidth observed here for growths on vicinal substrates, is similar to that previously reported for InGaAs quantum wells with GaAs barriers, which has been attributed to interfacial roughness and composition variations due to step-bunching growth. ${ }^{12}$ The lumi-
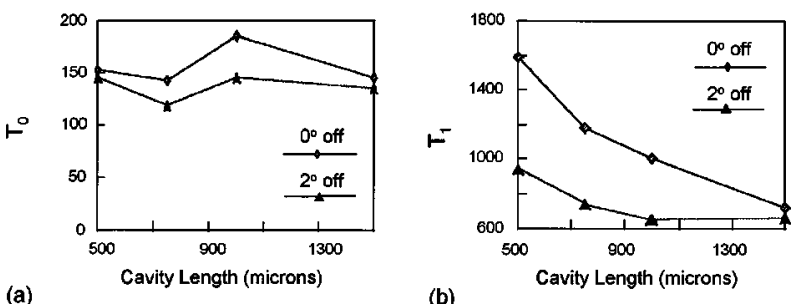

FIG. 2. Measured characteristic temperature coefficients: (a) $T_{0}$ of the threshold current, and (b) $T_{1}$ of the differential quantum efficiency, as a function of cavity length for lasers grown on (100) GaAs substrates $0^{\circ}$-, and $2^{\circ}$-off toward [110]. Significant improvement is found for devices grown on exact orientation (100) substrates.

nescence shift towards lower energy, observed in this work, for growths on misoriented substrates results either from a slightly larger quantum-well thickness compared with that on the on-orientation substrate growths, or from higher In incorporation in quantum wells with increased substrate misorientation. By contrast, luminescence was observed to shift towards higher energy for 6-nm-wide InGaAs/GaAs quantum wells grown on misoriented substrates, ${ }^{12}$ as a result of lower In incorporation in the quantum wells. In order to correlate the InGaAs/InGaAsP quantum well luminescence properties with device properties, the performances of laser structures grown on substrates oriented exact (100) and misoriented $2^{\circ}$ towards [110] were measured and compared.

The laser structure used in the device comparison study consists of an active region with two $70 \AA$ A-wide $\mathrm{In}_{x} \mathrm{Ga}_{1-x} \mathrm{As} \quad(x=0.18)$ QWs and a $100 \AA$-wide InGaAsP $\left(E_{g}=1.62 \mathrm{eV}\right)$ barrier, placed within an undoped 2000 $\AA$-wide InGaAsP $\left(E_{g}=1.62 \mathrm{eV}\right)$ confining region, which is sandwiched between $1.5 \mu \mathrm{m}$-thick InGaP cladding layers (Zn-doped: $10^{18} \mathrm{~cm}^{-3}$ and Si-doped: $10^{18} \mathrm{~cm}^{-3}$ ). The DQW active region lowers the quasi-Fermi level, and thereby reduces carrier leakage from the wells to the confinement layers, which along with the relatively high band-gap InGaAsP confinement layers results in strong carrier confinement to the active region. This reduces the temperature sensitivity of $\eta_{d}$, and allows high cw output powers to be obtained. $100-\mu \mathrm{m}$-wide stripe lasers were cut into various cavity lengths for testing under pulsed operation. A roomtemperature study of $I_{\mathrm{th}}$ and $\eta_{d}$ as a function of cavity length reveals no significant differences between laser structures grown on exact (100) and $2^{\circ}$ off towards [110]. Under pulsed operation, 1-mm long devices exhibit threshold-current densities of $140-160 \mathrm{~A} / \mathrm{cm}^{2}, \eta_{d}=65 \%-70 \%$, internal efficiency of $90 \%$, internal loss in the range $5-7 \mathrm{~cm}^{-1}$, and a transparency current density of $50 \mathrm{~A} / \mathrm{cm}^{2}$ for structures grown on both types of substrate orientation. However, significant differences are observed in the temperature sensitivity of both $I_{\text {th }}$ and $\eta_{d}$. The $I_{\text {th }}$ characteristic temperature coefficient $T_{0}$, and $\eta_{d}$ characteristic temperature coefficient $T_{1}$, where $T_{1}$ is defined as

$$
\eta_{d}=\eta_{d}\left(20^{\circ} \mathrm{C}\right) \exp \left[-\left(T-20^{\circ} \mathrm{C}\right) / T_{1}\right],
$$

with $T$ being the heatsink temperature, were measured over the temperature interval $20-55^{\circ} \mathrm{C}$, as a function of cavity length for laser structures grown on both orientated and mis- 


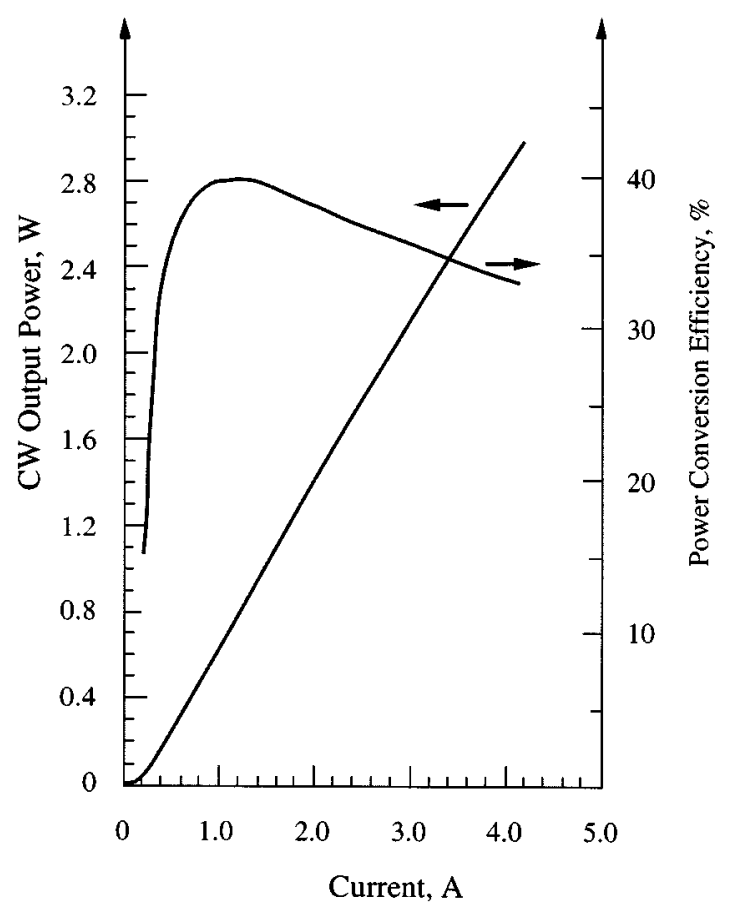

FIG. 3. cw output power (both facets) vs current characteristics for an uncoated DQW diode laser with dimensions $100 \mu \mathrm{m} \times 600 \mu \mathrm{m}$. The total power conversion efficiency $\eta_{p}$, reaches a maximum of $40.2 \%$ at $0.8 \mathrm{~W}$ and $8 \times$ lasing threshold. The series resistance is $0.12 \Omega$.

oriented substrates. The results, shown in Figs. 2(a) and 2(b), indicate significant improvement in the $T_{0}$, and $T_{1}$ for laser structures grown on exact (100) substrates. For 1-mm-long devices, lasers grown on exact (100) substrates exhibit high characteristic temperature coefficients for both threshold and differential quantum efficiency: $T_{0}=190{ }^{\circ} \mathrm{C}$, and $T_{1}$ $=1000{ }^{\circ} \mathrm{C}$. These values correspond to a $20 \%$ increase in $I_{\text {th }}$ and a $3.4 \%$ decrease in $\eta_{d}$ over the measured temperature range (i.e., $20-55^{\circ} \mathrm{C}$ ). In contrast, 1-mm-long lasers grown on $2^{\circ}$-misoriented substrates exhibit $T_{0}=145{ }^{\circ} \mathrm{C}$, and $T_{1}$ $=650{ }^{\circ} \mathrm{C}$, corresponding to a $27.3 \%$ increase in $I_{\text {th }}$ and a $5.3 \%$ decrease in $\eta_{d}$. The difference in $\eta_{d}$ temperature sensitivity is more marked for shorter devices. Thus, for 500$\mu \mathrm{m}$-long lasers, the relative decrease in $\eta_{d}$ over the 20$55^{\circ} \mathrm{C}$ temperature range is twice as much for $2^{\circ}$-off lasers than for $0^{\circ}$-off lasers. This happens in spite of the fact that $0^{\circ}$-off lasers emit at wavelengths $15 \mathrm{~nm}$ shorter than $2^{\circ}$-off lasers, ${ }^{11}$ and thus should have more carrier leakage than $0^{\circ}$ off lasers emitting at the same wavelength as $2^{\circ}$-off lasers (i.e., $0.96 \mu \mathrm{m}$ ). We conclude that at high temperatures carrier leakage and subsequent free-carrier absorption in the confinement layers is much more severe for $2^{\circ}$-off lasers than for $0^{\circ}$-off lasers because of interfacial imperfections caused by the "step-bunching" effect in misoriented-substrate devices.

Devices fabricated from laser structures grown on exact (100) substrates were mounted (uncoated facets) junctionside down on diamond heatsinks and measured under $\mathrm{cw}$ operation. The measured output power versus $\mathrm{cw}$ current for a device with a $600 \mu \mathrm{m}$ cavity length is shown in Fig. 3. The $3 \mathrm{~W}$ power level achieved represents the highest $\mathrm{CW}$ output power reported to date for $\mathrm{InGaAs} / \mathrm{InGaAsP} / \mathrm{InGaP} \mathrm{Al}$-free lasers. Catastrophic optical mirror damage for these uncoated devices occurs at a flux density of $\approx 5 \mathrm{MW} / \mathrm{cm}^{2}$. The measured series resistance $R_{s}$, is $0.12 \Omega$; that is $\approx 4$ times less than for AlGaAs-clad devices of similar contact-stripe geometry. ${ }^{16,17}$ The power conversion efficiency $\eta_{p}$, reaches a maximum value of $40.2 \%$ at $0.8 \mathrm{~W}$, maintains values above $35 \%$ from 0.25 to $2.4 \mathrm{~W} \mathrm{cw}$, and then decreases only to $33 \%$ at the maximum power of $3 \mathrm{~W}$. These consistently high $\eta_{p}$ values are the result of high $T_{0}$ and $T_{1}$ values, very low electrical resistance and high thermal conductivity of the InGaP cladding layers, and the use of a diamond heatsink. We note in particular that the $\eta_{p}$ maximum occurs at $8 \times I_{\text {th }}$, a value quite close to that calculated using theory ${ }^{18}$ (i.e., $9.5 \times I_{\text {th }}$ ) with the measured $R_{s}$ value. It is also worth noting that the record output power achieved here from uncoated devices is the same as the highest output power previously demonstrated ${ }^{19}$ from optimized facet-coated InGaAs/AlGaAs diode lasers with passivated mirror facets.

In conclusion, the growth of InGaAs/InGaAsP/InGaP QW structures has been studied with respect to substrate orientation. Growths on exact orientation substrates exhibit narrow linewidth PL and provide diode lasers of reduced temperature dependence of $I_{\text {th }}$ and $\eta_{d}$. DQW laser structures grown on exact (100) substrates have demonstrated recordhigh cw output powers.

${ }^{1}$ S. H. Groves, Z. L. Liau, S. C. Palmateer, and J. N. Walpole, Appl. Phys. Lett. 56, 312 (1989).

${ }^{2}$ C. Zmudzinski, D. Botez, L. J. Mawst, A. Bhattacharya, M. Nesnidal, and R. F. Nabiev, IEEE J. Special Topics Quantum Electron. 1, 129 (1995).

${ }^{3}$ J. Diaz, I. Eliashevich, K. Mobarhan, E. Kolev, L. J. Wang, D. Z. Garbuzov, and M. Razeghi, IEEE Photonics Technol. Lett. 6, 132 (1994).

${ }^{4}$ M. C. Wang, D. M. Hwang, P. S. D. Lin, L. Dechiaro, C. E. Zah, S. Ovadia, T. Lee, and D. Darby, Appl. Phys. Lett. 64, 3145 (1994).

${ }^{5}$ J. M. Olson, R. K. Ahrenkiel, D. J. Dunlavy, B. Keyes, and A. E. Kibbler, Appl. Phys. Lett. 55, 1208 (1989).

${ }^{6}$ D. Z. Garbuzov, N. Y. Antonishkis, A. D. Bondarev, S. Z. Zhigulin, A. V. Kochergin, N. I. Katsavets, and E. U. Rafailov, IEEE J. Quantum Electron. QE-7, 1531 (1991).

${ }^{7}$ E. C. Vail, R. F. Nabiev, and C. J. Chang-Hasnain, IEEE Photonics Technol. Lett. 6, 1303 (1994).

${ }^{8}$ M. Sagawa, T. Toyonaka, K. Hiramoto, K. Shinoda, and K. Uomi, IEEE J. Selected Topics Quantum Electron 1, 189 (1995).

${ }^{9}$ H. Asonen, A. Ovtchinnikov, G. Zhang, J. Nappi, P. Savolainen, and M. Pessa, IEEE J. Quantum Electron. QE-30, 415 (1994).

${ }^{10}$ M. Ohkubo, T. Ijichi, A. Iketani, and T. Kikuta, IEEE J. Quantum Electron. QE-30, 408 (1994).

${ }^{11}$ L. J. Mawst, A. Bhattacharya, M. Nesnidal, J. Lopez, D. Botez, J. A. Morris, and P. Zory, Electron. Lett. 31, 1153 (1995).

${ }^{12}$ K. Hiramoto, T. Tsuchiya, M. Sagawa, and K. Uomi, J. Cryst. Growth 145, 133 (1994).

${ }^{13}$ D. Bour, R. S. Geels, D. W. Treat, T. L. Paoli, F. Ponce, R. L. Thornton, B. S. Krusor, R. D. Bringans, and D. F. Welch, IEEE J. Quantum Electron. QE-30, 593 (1994).

${ }^{14}$ M. Jou, J. Lin, C. Chang, C. Lin, M. Wu, and B. Lee, Jpn. J. Appl. Phys. 32, 4460 (1993).

${ }^{15}$ D. Grutzmacher, J. Hergeth, F. Reinhardt, K. Wolter, and P. Balk, J. Electron Mater. 19, 471 (1990).

${ }^{16}$ D. P. Bour, G. A. Evans, and D. B. Gilbert, J. Appl. Phys. 65, 3340 (1989).

${ }^{17}$ N. W. Carlson (private communication).

${ }^{18}$ R. G. Waters, D. K. Wagner, D. S. Hill, P. L. Tihanyi, and B. J. Vollmer, Appl. Phys. Lett. 51, 1318 (1987).

${ }^{19}$ D. F. Welch, W. Streifer, C. F. Schaus, S. Sun, and P. L. Gourley, Appl. Phys. Lett. 56, 10 (1990). 